\title{
Three Sequential Balloon Catheters for Vascular Exclusion of the Liver and Aortic Control (one REBOA and two REBOVCs): A Hemorrhage Control Strategy in Suprahepatic Vena Cava Injuries
}

\author{
1 Joao B Rezende-Neto, ${ }^{2}$ Ghassan Al-Kefeiri, ${ }^{3}$ Matt Strickland, ${ }^{4}$ Vikram Prabhudesai, ${ }^{5}$ Sandro B Rizoli, ${ }^{6}$ Ori Rotstein
}

\begin{abstract}
Introduction: We hypothesized that sequential deployment of a resuscitative endovascular balloon occlusion in the aorta REBOA, Pringle maneuver, and two Resuscitative balloon occlusion of the inferior vena cava (REBOVC) would provide hepatic vascular exclusion and hemorrhage control.
\end{abstract}

Materials and Methods: Hemodynamic monitoring and splenectomy were performed in seven swine. One REBOA device and two REBOVCs were positioned under fluoroscopy in the thoracic aorta, suprahepatic and infrahepatic inferior vena cava (IVC); $35 \%$ of the total blood volume was removed. Hepatic vascular exclusion was performed for 15 minutes during shock through sequential deployment of the REBOA in the thoracic aorta, Pringle maneuver, the REBOVC in the infrahepatic IVC, and the REBOVC in the suprahepatic IVC. Hepatic vascular exclusion was reversed in the following sequence: Deflation of the REBOVC in the suprahepatic IVC, followed by the infrahepatic IVC REBOVC, release of the Pringle, and deflation of the REBOA. Subsequently, a $1.5 \mathrm{~cm}$ injury was performed in the suprahepatic IVC. Immediately thereafter, hepatic vascular exclusion was performed for 15 minutes followed by reversal of exclusion; suture repair of the injury was performed in two animals.

Results: Hepatic vascular exclusion effectively stopped the bleeding from the suprahepatic IVC injury, significantly increased MAP. The procedure did not aggravate shock assessed by $\mathrm{pH}$, lactate and base excess, and hemodynamics. Reversal of the exclusion led to immediate exsanguination from the suprahepatic IVC injury except after suture repair of the injury.

${ }^{1}$ Associate Professor of Surgery, ${ }^{2}$ Research Fellow, ${ }^{3}$ Resident in General Surgery, ${ }^{4}$ Staff Radiologist, ${ }^{5,6}$ Professor of Surgery

${ }^{1}$ Department of Surgery, St. Michael's Hospital, University of Toronto, Toronto, Ontario, Canada

2,3,5,6 Surgery, University of Toronto and Division of General Surgery St. Michael's Hospital, Toronto, Ontario, Canada

${ }^{4}$ Department of Medical Imaging, St. Michael's Hospital, Toronto, Ontario, Canada

Corresponding Author: Joao B Rezende-Neto, Associate Professor of Surgery, Department of Surgery, St. Michael's Hospital, University of Toronto, Toronto, Ontario, Canada, Tel:6472900404, email: rezendenetoj@smh.ca
Conclusion: Sequential deployment of REBOA, Pringle maneuver and two REBOVCs provided vascular exclusion of the liver and effectively temporized the hemorrhage from the suprahepatic IVC.

Clinical Significance: Vascular exclusion of the liver during operative repair is difficult in the setting of massive bleeding. The procedure described herein is less invasive and effectively controls the bleeding.

Keywords: Endovascular balloon; Hemorrhage control, Suprahepatic; Vena cava injury.

How to cite this article: Rezende-Neto JB, Al-Kefeiri G, Strickland M, Prabhudesai V, Rizoli SB, Rotstein O, Three Sequential Balloon Catheters for Vascular Exclusion of the Liver and Aortic Control (one REBOA and two REBOVCs): A Hemorrhage Control Strategy in Suprahepatic Vena Cava Injuries. Panam J Trauma Crit Care Emerg Surg 2018;7(2):114-122.

Source of support: Nil

Conflict of interest: None

\section{INTRODUCTION}

Injuries to the retrohepatic and the suprahepatic segments of the inferior vena cava have mortality rates over $80 \%$. $^{1,2}$ Treatment of these require expeditious mobilization of the liver for clear exposure of the retrohepatic and the suprahepatic inferior vena cava. This move is technically difficult to carry out in the presence of active bleeding increasing the risk of rapid intraoperative exsanguination even when performed by seasoned trauma surgeons. Moreover, because patients are usually hemodynamic unstable on presentation any attempt to occlude the inferior vena cava below the liver, where it is easily accessible, will provoke reduction in preload and sudden cardiovascular collapse. Furthermore, Pringle maneuver and rigorous packing of the liver are not effective hemorrhage control strategies for treating injuries to the retrohepatic and the suprahepatic inferior vena cava. ${ }^{2,3}$ Unfortunately, additional approaches aimed at temporizing hemorrhage from retrohepatic and suprahepatic inferior vena cava injuries proved to be unsatisfactory, only scantly improving survival rates by 25 to $30 \%$. 1,3

Case in point, the atriocaval shunt (Schrock shunt) is a technically challenging procedure and is linked to 
significant complications. ${ }^{4,5}$ Consequently, it is often used as a "last ditch" recourse to control bleeding in patients with retrohepatic inferior vena cava (RH-IVC) and suprahepatic inferior vena cava (SH-IVC) injuries. Moreover, the atriocaval shunt does not provide complete vascular isolation of the liver. Intravascular balloon shunt inserted in the IVC via the saphenousfemoral junction is a less invasive option to provide hemorrhage control and venous return prior to surgical exploration of the injury. ${ }^{6,7}$ However, comprehensive usage of this method has not been reported. Venovenous bypass as an adjunct to hepatic vascular exclusion has also been used sporadically with variable success. ${ }^{8-11}$ Ultimately, nonoperative management of injuries to the retrohepatic and the suprahepatic IVC has also been described. However, the success of this strategy is for the most part unpredictable, despite multidisciplinary approach involving interventional radiologic techniques. ${ }^{4,5,12,13}$

Complete vascular exclusion of the liver during direct operative repair (Heaney maneuver) provides proximal and distal control of the IVC, portal inflow occlusion, and a potentially "bloodless field". This approach was used in combination with selective aortic cross-clamping in a series of 10 patients with juxtahepatic IVC injuries resulting in a survival rate of $70 \% .{ }^{14}$ Notwithstanding its suitability, intra-operative vascular exclusion of the liver by sequential occlusion of the IVC and the hepatic pedicle is a difficult procedure to perform in the setting of massive bleeding. Therefore, hemorrhage control through an endovascular route is an appealing strategy prior to direct repair of RH-IVC and SH-IVC injuries. The emergence of REBOA has spawned interest in the use of this technology to control hemorrhage in major abdominal venous injury. ${ }^{15-19}$ Two recent studies describe the use of REBOVC to control bleeding from this vessel. ${ }^{18,20}$ However, the animal model used in the most current study did not faithfully simulate the uncontrolled nature of an injury to the suprahepatic IVC in the setting of pre-existing hemorrhagic shock.

In the present study, we describe a technique for complete hepatic vascular exclusion and aortic occlusion with sequential deployment of three (REBOA) and two in the vena cava REBOVC in addition to the Pringle maneuver. This approach can be used that can be used both intra-operatively and pre-operatively.

\section{MATERIALS AND METHODS}

This study was approved by the Animal Care Committee of the Li Ka Shing International Knowledge Institute of St. Michael's Hospital (protocol number ACC 629). A total of seven adult male Yorkshire swine (Sus scrofa) weighing $56.5 \mathrm{~kg}( \pm 6.5 \mathrm{~kg})$ were acclimated for 7 days prior to the experiment.

\section{Animal Preparation}

In brief, animals were anesthetized with ketamine (20 mg/ kg intramuscular injection), xylazine ( $2 \mathrm{mg} / \mathrm{kg})$, and atropine sulphate $(1 \mathrm{mg} / 25 \mathrm{~kg})$, followed by intubation. Mechanical ventilation was set at tidal volume of $10 \mathrm{ml} / \mathrm{kg}$ and a respiratory rate of 15 breaths per minute. Anesthesia was maintained throughout the procedure with Isoflurane inhalation (2-5\%). Pulse oximetry, electrocardiogram (ECG), and heart rate were monitored continuously; animals were placed on a warming blanked at $39^{\circ} \mathrm{C}$. The left carotid artery, the right femoral artery, and the left internal jugular vein were cannulated with 7 Fr sheaths (Super Sheath ${ }^{\mathrm{TM}}$, Boston Scientific Corporation, Natick, MA) for monitoring of mean arterial blood pressure (MAP), blood collection, fluid infusion, and central venous pressure (CVP). Baseline laboratory tests, MAP, and CVP were obtained at this point. A guidewire was introduced in the left femoral artery (LFA) by a countdown approach. The wire was advanced into the thoracic aorta under fluoroscopic guidance (OEC Brivo Plus, General Electric Healthcare, Wauwatosa, WI). Subsequently, the first 10Fr REBOACODA $®$ balloon catheter (Cook Inc., Bloomington, IN) was advanced over the guidewire. Proper positioning of the $40 \mathrm{~mm}$ balloon within the aorta and complete aortic occlusion were confirmed by loss of the arterial waveform from the right femoral artery by briefly inflating the balloon with 7cc of iodinated contrast media (OMNIPAQUE 240, Sanofi-Winthrop, New York, NY). Afterward, the second 10Fr CODA ${ }^{\circledR}$ balloon catheter was inserted in the suprahepatic IVC through a cutdown in the right femoral vein under fluoroscopic guidance. Proper positioning of the $40 \mathrm{~mm}$ balloon just above the diaphragm within the suprahepatic IVC was verified by briefly inflating the balloon with iodinated contrast media (Fig. 1). Ultimately, the left groin was explored to expose the left femoral vein. The third CODA ${ }^{\circledR}$ balloon catheter $(10 \mathrm{Fr})$ was introduced in the left femoral vein as previously described. The balloon was advanced into the infrahepatic IVC and positioned within the IVC just above the renal veins under fluoroscopic guidance. A proximal ligature (2-0 silk suture-SOFSILK ${ }^{\mathrm{TM}}$, Covidien, Sunnyvale, CA) was placed around each vessel proximal to the point of entry of the balloon catheter. Thus, preventing catheter dislodgement. To prevent dislodgment, each catheter was secured in place with a proximal ligature around the corresponding vessel and the catheter using a Positioning of the balloon catheters, the MAP 


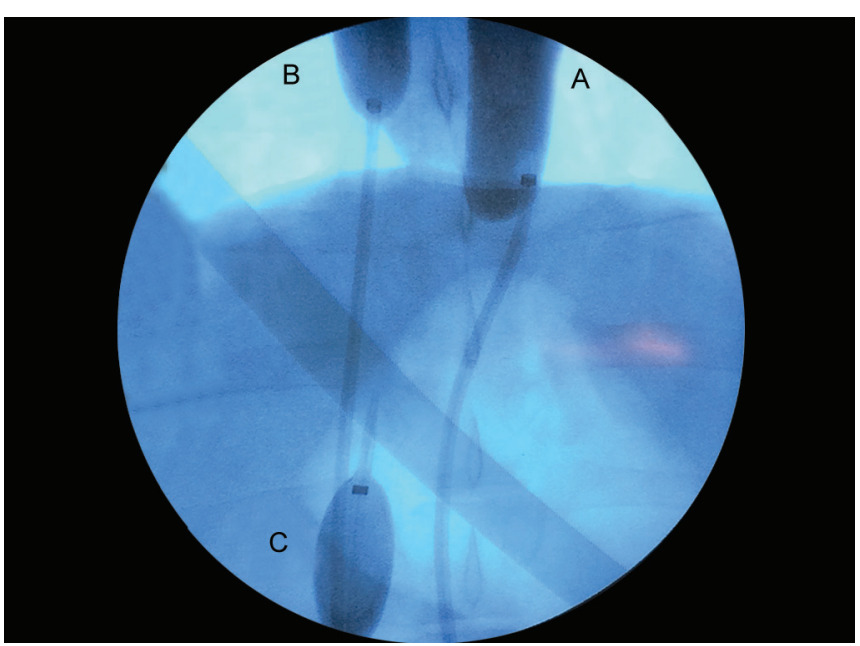

Fig. 1: Fluoroscopic image of the endovascular balloons inflated within the aorta (A) in the suprahepatic IVC; $(B)$ and the infrahepatic IVC; (C) IVC, inferior vena cava.

and the CVP were reassessed following a five-minute observation period. Subsequently, a splenectomy was performed through a midline laparotomy to prevent splenic contraction and hemodynamic variation from autotransfusion. A $500 \mathrm{ml}$ bolus of Ringer's lactate was given at this time and hemodynamics reassessed.

\section{Hemorrhagic Shock}

Shock was induced by hemorrhage from the catheter sheath positioned in the left carotid artery. The target hemorrhage volume was set at $35 \%$ of the total blood volume in adult swine $(66 \mathrm{ml} / \mathrm{Kg})$ as described in previous research. ${ }^{21}$ Hemorrhage lasted for 20 minutes, with half of the target volume removed during the first 7 minutes and the remainder removed during the last 13 minutes as described in previous research. ${ }^{21}$ The withdrawal of blood was interrupted if MAP decreased to $<30 \mathrm{~mm} \mathrm{Hg}$, restarting hemorrhage when MAP increased above $30 \mathrm{mmHg}$. Blood was collected in a graduated glass jar (Chemglass Life Sciences, Vineland, NJ) containing 10,000 units of heparin. Animals were maintained in shock without any intervention for another 20 minutes after the target hemorrhage volume was removed. A new set of blood samples was obtained at the end of the 20-minute period.

\section{Total Vascular Exclusion of the Liver During Shock Without Suprahepatic IVC Injury}

The balloon within the thoracic aorta (REBOA) was inflated first. Subsequently, a Pringle maneuver was performed using a Satinsky clamp. Afterward, the balloon of the first REBOVC device was inflated within the infrahepatic IVC as previously described. Ultimately, the balloon of the second REBOVC device was inflated in the suprahepatic IVC. All balloons were inflated with
$7 \mathrm{ml}$ of iodinated contrast media. Proper positioning of the balloons were confirmed by fluoroscopy. Loss of the arterial waveform from the right femoral artery confirmed complete aortic occlusion. Total vascular exclusion of the liver was confirmed by absence of flow within the liver parenchyma through intra-operative Doppler ultrasonography. The balloons were maintained inflated concurrently with Pringle maneuver for a period of 15 minutes. Hemodynamic data were recorded throughout this period. Subsequently, hepatic vascular exclusion was reversed through balloon deflation and release of the Pringle maneuver in the following sequence: first, the deflation of the REBOVC device positioned within the suprahepatic IVC; secondly, deflation of the REBOVC device positioned within the infrahepatic IVC followed by release of the Pringle maneuver, and finally deflation of the REBOA device positioned within the thoracic aorta. A bolus of $1 \mathrm{~L}$ of Ringer's lactate was given at this time and balloons were maintained deflated for an additional 30 minutes.

\section{Total Vascular Exclusion of the Liver with Suprahepatic IVC Injury}

The objective in this phase of the experiment was to investigate hemorrhage control using the three balloons and the Pringle maneuver in the presence of a standardized injury of the SH-IVC. The right lobe of the liver was partially mobilized to expose a small segment of the SH-IVC. Subsequently, a $1.5 \mathrm{~cm}$ longitudinal injury was created along the anterior wall of the SH-IVC with a number 11 surgical blade (Integra Miltex Medical Instruments, York, PA.) The injury extended caudally towards the liver beginning immediately above the confluence of the hepatic veins (Fig. 2). Profuse bleeding ensued from the injury immediately. Manual

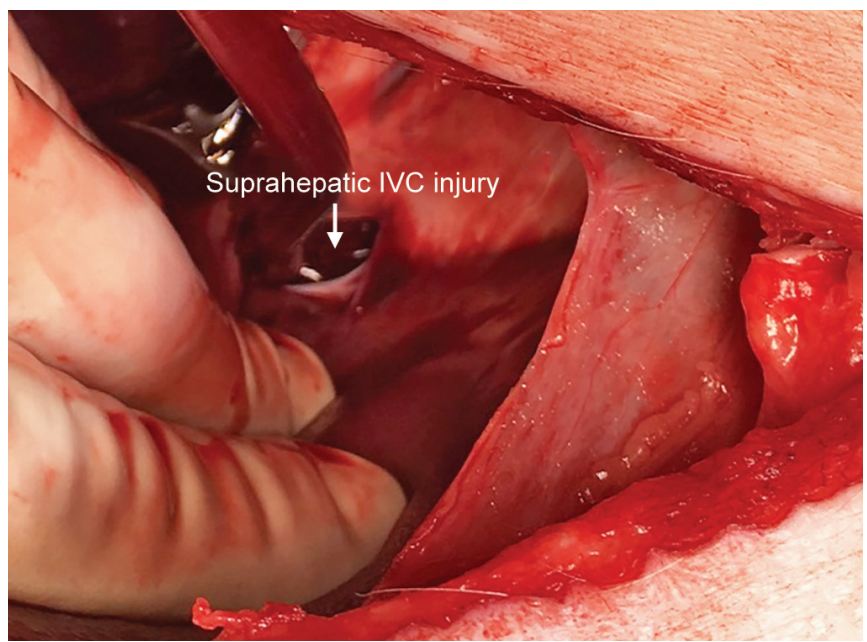

Fig. 2: Intraoperative photograph depicting the injury $(1.5 \mathrm{~cm})$ to the anterior wall of the suprahepatic IVC (arrow). IVC, inferior vena cava. 
compression was briefly applied to the liver followed by hepatic vascular exclusion through sequential deployment of the REBOA device, Pringle maneuver, the REBOVC device positioned within the infrahepatic IVC, and lastly the REBOVC device positioned within the suprahepatic IVC (Fig. 3). Total vascular exclusion of the liver for hemorrhage control continued for 15 minutes. Hemodynamic data were recorded throughout this period and blood samples were obtained for laboratory tests. Reversal of hepatic vascular exclusion was performed at the end of the 15-minute period. The balloons were sequentially deflated and the Pringle maneuver was released as previously described (Fig. 4). This move resulted in profuse bleeding from the injury site on the anterior wall of the SH-IVC causing the animals to die from exsanguination shortly thereafter.

In two animals the injury was repaired with running 5-0 polypropylene sutures (ETHICON, Somerville, NJ) prior to balloon deflation to ascertain hemorrhage control after balloon deflation.

Data were analyzed using GraphPad Prism version 6.00 for Windows (GraphPad Software, La Jolla, CA). Hemodynamic and laboratory data are reported as mean \pm SD. Student's $t$ tests were performed for continuous variables between two interventions/time points, analysis of variance ANOVA for comparison of three or more interventions/variables/time points and Tukey's

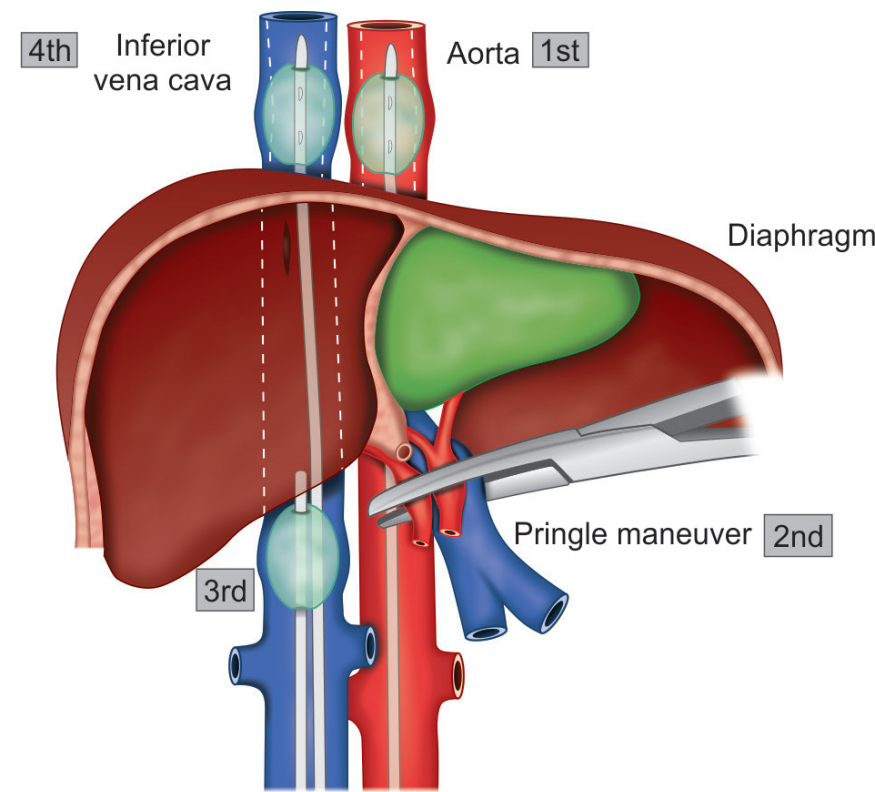

Fig. 3: Complete vascular exclusion of the liver. In sequence, deployment of the REBOA device (1st), Pringle maneuver (2nd), deployment of the first REBOVC positioned in the infrahepatic

IVC, above the renal veins (3rd), deployment of the second REBOVC in the suprahepatic IVC (4th). REBOA, Resuscitative endovascular balloon occlusion of the aorta; REBOVC, Resuscitative endovascular balloon occlusion of the inferior vena cava; IVC, Inferior vena cava.

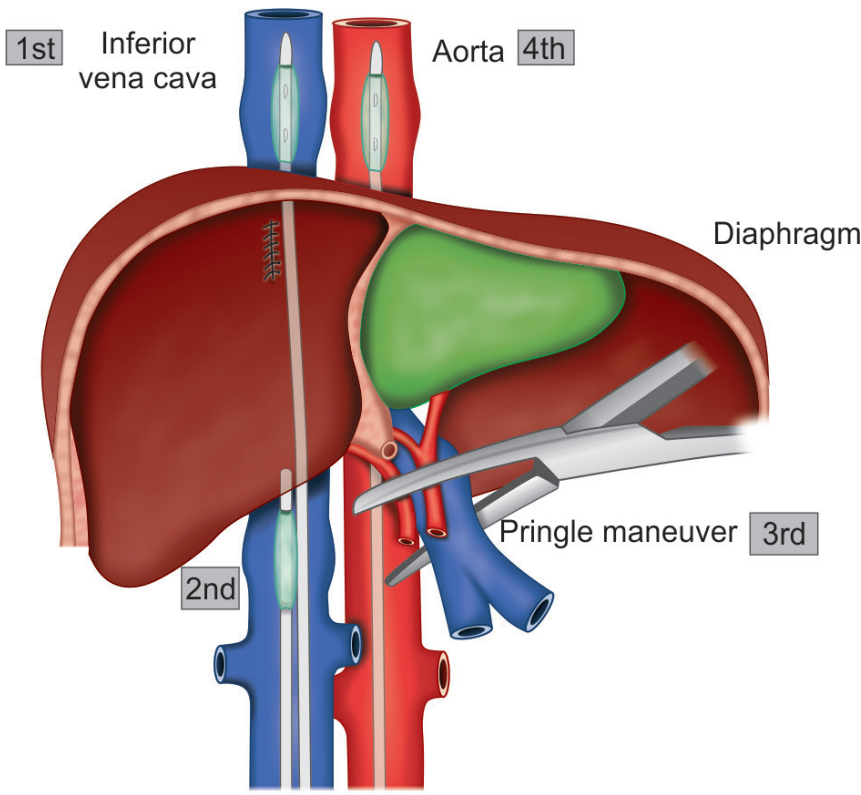

Fig. 4: Reversal of vascular exclusion of the liver. In sequence, deflation of the REBOVC positioned in the suprahepatic IVC (1st), deflation of the REBOVC positioned in the infrahepatic IVC (2nd), release of Pringle maneuver (3rd), deflation of the REBOA device (4th). REBOVC, resuscitative endovascular balloon occlusion of the inferior vena cava; IVC, inferior vena cava; REBOA, resuscitative endovascular balloon occlusion of the aorta.

multiple comparison test; $\mathrm{p}<0.05$ was considered statistically significant.

\section{RESULTS}

Functionally, the endovascular balloon catheters and the Pringle maneuver effectively halted the bleeding from the injury to the SH-IVC. Moreover, the two animals that underwent suture of the injury did not bleed after balloon deflation.

Hemorrhagic shock resulted in significant hypotension, the nadir in MAP occurred at 20 minutes (Fig. 5). Vascular exclusion of the liver through deployment of the REBOA device followed by Pringle maneuver, and the REBOVC devices augmented MAP to levels equivalent to those seen during the surgical preparation phase. However, statistically significant reduction in MAP ensued despite an intact SH-IVC upon balloon deflation and release of the Pringle maneuver during reversal of hepatic vascular exclusion (Table 1). Interestingly, the hemodynamic response to hepatic vascular exclusion in the setting of SH-IVC injury was similar to that of hepatic vascular exclusion without injury to the SH-IVC. However, reversal of the hepatic vascular exclusion in the presence of the SH-IVC injury resulted in rapid exsanguination. This event led to drastic decrease in MAP and CVP (Table 1). A significant decrease in heart rate ensued and shortly thereafter, the animal expired from the bleeding. 


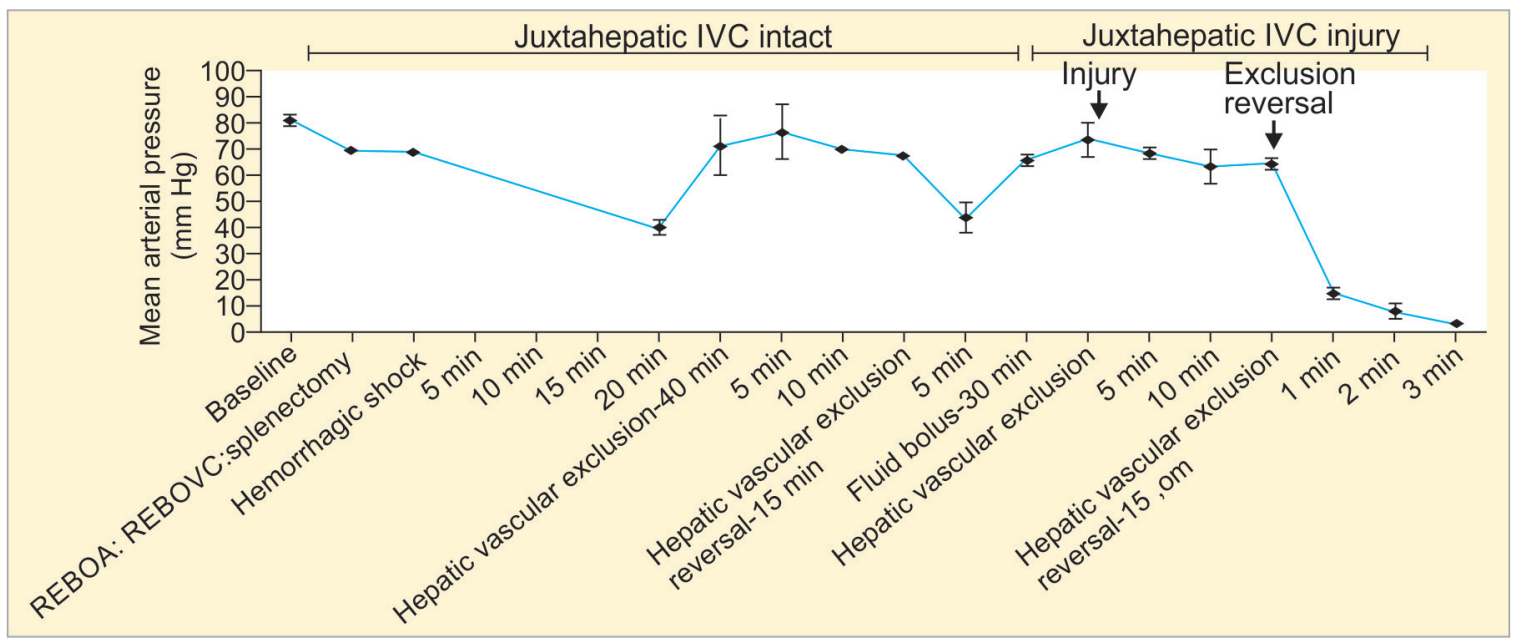

Fig. 5: Experimental design and hemodynamic response. Mean arterial pressure measured in the left carotid artery.

Table 1: Hemodynamic response to shock, hepatic vascular exclusion, suprahepatic IVC Injury, and reversal of hepatic vascular exclusion

\begin{tabular}{|c|c|c|c|c|c|c|}
\hline Parameters & Baseline & $\begin{array}{l}\text { Shock (20 } \\
\text { min) }\end{array}$ & $\begin{array}{l}\text { Hepatic vascular } \\
\text { exclusion } \\
\text { (SH-IVC intact) }\end{array}$ & $\begin{array}{l}\text { Hepatic vascular } \\
\text { exclusion } \\
\text { (SH-IVC injury) }\end{array}$ & $\begin{array}{l}\text { Deflation/ } \\
\text { pringle release } \\
\text { (SH-IVC injury) }\end{array}$ & $p$ \\
\hline HR (bpm) & $102 \pm 13$ & $111 \pm 27$ & $116 \pm 17$ & $132.6 \pm 20.6$ & $72.4 \pm 34.5$ & $0.011^{*}$ \\
\hline MAP (mm Hg) & $80.5 \pm 3.9$ & $40 \pm 3.7$ & $68.4 \pm 3.8$ & $66 \pm 5.8$ & $10 \pm 7.5$ & $<0.001 \dagger$ \\
\hline CVP (mm Hg) & $7.4 \pm 2.1$ & $5.6 \pm 1.5$ & $6.4 \pm 1.1$ & $6.0 \pm 1.0$ & $1.0 \pm 1.4$ & $<0.0001 \ddagger$ \\
\hline
\end{tabular}

IVC-Inferior vena cava; SH-Suprahepatic, HR-Heart rate; MAP-Mean arterial pressure; CVP-Central venous pressure *1 min after reversal of hepatic vascular exclusion vs. all other interventions. $\dagger 1 \mathrm{~min}$ after reversal of hepatic vascular exclusion vs. all other interventions; Shock 20 minutes vs. baseline and all hepatic vascular exclusion; baseline vs. all hepatic vascular exclusion. $¥ 1$ min after reversal of hepatic vascular exclusion vs. all other interventions; analysis of variance with Tukey's multiple comparison test; values represent mean $\pm \mathrm{SD} ; \mathrm{p}<0.05$ statistically significant.

Laboratory tests performed 20 minutes after the beginning of shock showed significant reduction $(\mathrm{p}<0.05)$ in the following parameters compared to baseline: $\mathrm{pH}(7.5 \pm 0.1$ vs. $7.2 \pm 0.5)$, cBase extracellular fluid $(2.6 \pm 1.1$ vs. $-4.8 \pm 2.3 \mathrm{mmol} / \mathrm{L})$, and hemoglobin $(122.8 \pm 8.5$ vs. $65.2 \pm 10.6 \mathrm{~g} / \mathrm{L})$. Moreover, hemorrhagic shock also provoked an increase in serum lactate $(1.8 \pm 0.4$ vs. $5.8 \pm 1.2 \mathrm{mmol} / \mathrm{L}$ ). Laboratory tests performed 15 minutes after complete vascular exclusion of the liver in the presence of SH-IVC injury were similar to the results observed during shock alone; respectively $\mathrm{pH}(7.2 \pm 0.5 \mathrm{vs} .7 .3 \pm 0.3)$, cBase extracellular fluid $(-4.8 \pm 2.3$ vs. $-3.5 \pm 3.7 \mathrm{mmol} / \mathrm{L})$, and hemoglobin (65.2 \pm 10.6 vs. $64.2 \pm 10.5)$; $(\mathrm{p}>0.05)$.

\section{DISCUSSION}

Control of noncompressible torso hemorrhage with intra-aortic balloon occlusion catheter was initially described in the early 1950s. ${ }^{22,23}$ Recent studies have shown that REBOA is a safe and effective procedure for temporizing hemorrhage during the acute resuscitation phase of uncontrolled traumatic bleeding below the diaphragm. ${ }^{17,19,21,24-28}$ The notion that an endovascular balloon device could be used to temporize hemorrhage from injuries to the SH-IVC is not new. Several variations of this concept have been reported. ${ }^{6,79,16,18,29-31}$ However, the ideal method to achieve total vascular exclusion of the liver using endovascular balloon technology has not been stablished in the acute setting. $6,11,14,16,18,20,31,32$

In the present study, we describe the use of three endovascular balloon catheters in complete vascular exclusion of the liver as an alternative to direct surgical cross-clamping of the vessels. Our findings showed that this was an effective strategy to control bleeding from the suprahepatic segment of the IVC. Actually, reversal of the hepatic vascular exclusion in the setting of an injury to the SH-IVC resulted in almost instant exsanguination. Whereas reversal of hepatic vascular exclusion after suture repair of the injury (2 animals) did not result in bleeding.

Although, control of the infrahepatic cava and the Pringle manuever are relatively easy procedures to perform during a laparotomy, seasoned surgeons know that neither would provide effective hemorrhage control in suprahepatic IVC injury. ${ }^{18}$ Our procedure could be used when an injury to the suprahepatic IVC is suspected intra-operatively and pre-emptively in the rare situation when the injury is detected in preoperative computed tomography scans.

The use of three balloons in the sequence that we describe herein effectively addressed the main challenges 
posed by suprahepatic IVC injuries. Initially, hemodynamic stability was achieved by inflation of the balloon positioned in the aorta REBOA. Subsequently, the Pringle maneuver and deployment of two REBOVCs provided complete vascular exclusion of the liver and a "bloodless" operative field. This sequence finds support in previous reports where aortic occlusion prior to a laparotomy, in the setting of massive intraabdominal bleeding, could potentially prevent sudden cardiovascular collapse. ${ }^{9,21,24,26,28,32-34}$ Moreover, experimental data also showed that zone I REBOA resulted in lower fluid and vasopressor requirements, and less acidosis compared to aortic cross-clamping through thoracotomy during shock resusucitation. ${ }^{20}$ Notwithstanding these findings, previous studies showed that complete occlusion of the aorta can be poorly tolerated during hemorrhagic shock. . $^{19,35,36}$ However, this adverse effect could be attenuated by performing only a partial occlusion of the aorta with the endovascular balloon. ${ }^{19,27}$ This strategy resulted in increased survival time and better physiologic response compared to complete REBOA, in a swine model of liver injury. ${ }^{19}$

In a recent experimental study, the use of a single REBOCV inflated in the suprahepatic IVC, Pringle maneuver, and direct control of the infrahepatic IVC with Rummel tourniquet (study group) was compared with the same procedures except for suprahepatic REBOVC inflation (control group) in animals with suprahepatic IVC injury. The authors did use REBOA device or cross clamp the aorta in any of the animals. Although the study group (suprahepatic REBOVC inflation) showed less bleeding and prolonged time to death the difference in outcomes were marginal. ${ }^{18}$ In contrast to our study where the animals were hypovolemic even before the injury to the suprahepatic IVC was created and autotransfusion was countered by prior splenectomy, the authors of that study performed supra-hepatic IVC injury under normovolemia and intact spleen. We hypothesize that these reasons explain the prolonged survival time of the animals in that study and the lack of vascular collapse despite infrahepatic IVC control with Rummel tourniquet, REBOVC inflation in the suprahepatic IVC and Pringle maneuver.

More recently, the concept of automated endovascular variable aortic control (EVAC) was described in a liver injury model using an extracorporeal circuit. ${ }^{37}$ This approach helped to prevent excessive aortic pressures above the REBOA while maintaining distal aortic blood flow. ${ }^{37}$ Similar strategy could be adapted to our method of vascular exclusion of the liver to prevent intravascular volume overload above the diaphragm. Interestingly, however, in the present study total occlusion of the aorta by complete inflation of the REBOA device did not provoke increase in the CVP compared to the shock phase and baseline values. This finding could be partially explained by pre-existing hypovolemia secondary to shock and the decrease in preload caused by concomitant occlusion of the IVC. ${ }^{24,28,32,36}$

Our study also showed that adequate hemodynamic response continued for the duration of the complete vascular exclusion of the liver in the setting of hemorrhagic shock. We attributed this finding to the occlusion of the aorta REBOA before the inflation of the balloons in the IVC REBOVCs. This sequence could have attenuated the detrimental impact caused by preload reduction and mesenteric pooling. Correspondingly, previous studies showed that complete vascular exclusion of the liver during elective hepatectomies resulted in a $52 \%$ reduction in the cardiac index and a decrease in mean arterial pressure of $14 \%$, despite preemptive infusion of crystalloid prior to the occlusion. ${ }^{38,39}$ However, when hepatic vascular exclusion was performed in combination with supra celiac cross-clamping of the aorta, the reduction in cardiac index was only $10 \%$ less and accompanied by an increase of $30 \%$ in the MAP. ${ }^{39}$ Moreover, it has been shown that aortic occlusion prior to hepatic vascular exclusion decreases pooling of blood in the splanchnic circulation and in the lower extremities. ${ }^{40}$

An additional advantage of our method is that it facilitates repositioning of the REBOA and the REBOVC devices, and the degree of occlusion of the aorta and the IVC based on the hemodynamic response of the patient. ${ }^{19,37}$ This recourse could be useful during resuscitation before a laparotomy, as well as intraoperatively. Actually, recent studies reported on bedside strategies to rapidly determine the inflation volume and the anatomic position of the balloon within the aorta using ultrasound and smartphone-based infrared imaging technology. ${ }^{41,42}$

In our study, we observed brief bleeding from the SH-IVC injury immediately after the deployment of the balloons during vascular exclusion of the liver. This bleeding, however, was negligible and had no detrimental effect on the hemodynamic status of the animals. Previous reports showed that this finding could be attributed to the residual blood trapped within the excluded segment of the IVC and in the liver parenchyma during hepatic vascular exclusion. ${ }^{36,39-41}$ Accordingly, intraoperative Doppler ultra-sonography performed during hepatic vascular exclusion showed no blood flow to the liver in our model.

The duration of complete vascular exclusion of the liver in our study was in keeping with previous reports that showed adequate tolerance to aortic cross-clamping and hepatic vascular exclusion during periods of 15 to 20 minutes. ${ }^{11,14,38,43-45}$ Moreover, from a pragmatic 
standpoint, previous studies showed that generally no more than 15 to 30 minutes are needed for suture repair of injuries to the RH-IVC and SH-IVC once hepatic vascular exclusion is achieved..$^{3,11,16,38,44-46}$ Interestingly, recent results from experimental and clinical research reported an average duration of aortic occlusion with REBOA devices between 15-40 minutes. ${ }^{19,24-26,28,36,43,47}$ Notably in our findings, laboratory results and hemodynamic parameters during the second hepatic vascular exclusion remained similar to the first hepatic exclusion, despite the injury to the RH-IVC and a total shock time duration of approximately 60 minutes. This finding underscores that the vascular exclusion procedure was well tolerated by the animals.

This study has important limitations mainly linked to the experimental model. Considering that the study was primarily designed for proof of concept, we used the fewest number of animals necessary to demonstrate the procedure. A formal control group was not included. Additionaly, a control group without balloon exclusion would invariably lead to immediate death of all the animals, hence an unnecessary investigation. Moreover, we simulated a worse-case scenario and did not incorporate current principles of hemorrhagic shock resuscitation pertaining to the use of vasopressors and blood products. We did not record the time to perform the procedure. Although placement of three balloon catheters through cutdown could potentially be time consuming, the procedure can be simplified using a percutaneous approach. Particularly with newer low profile REBOA catheters such as the wire free 7Fr. sheath ER-REBOA ${ }^{\mathrm{TM}}$ (Prytime Medical Devices Inc. Boerne, TX). Moreover, the aortic balloon catheter is the first one deployed as per the technique. Thus, instant hemodynamic improvement is achieved with the inflation of the balloon, providing hemodynamic support during the placement of the other two balloon catheters in the vena cava. Cardiovascular monitoring relied only on the assessmemt of MAP and CVP, which precluded the acquisition of more detailed information related to the hemodynamic response during total vascular exclusion of the liver. Lastly, we did not address the insertion of REBOVC devices through the internal jugular vein. This could be an alternate route for hepatic vascular exclusion when using our technique in the setting of pelvic fracture with a large pelvic hematoma.

In conclusion, sequential deployment one REBOA device and two REBOVCs associated with the Pringle maneuver enabled total vascular exclusion of the liver. This approach is less invasive than direct operative control of bleeding from injuries to the retrohepatic and suprahepatic IVC. Nevertheless, potential complications inherent to vascular access for balloon placement is a genunine concern. However, newer devices with smaller diameter could reduce these complications and expand the indications of the procedure as a preemptive measure in unstable patients who present with severe liver injuries.

\section{ACKNOWLEDGMENTS}

This work was funded by the Academic Enrichment Fund from the Division of General Surgery St. Michael's Hospital.

The authors are thankful to Danielle Gifford (Registered Veterinary Technician) for her assistance in the experiment.

\section{CLINICAL SIGNIFICANCE}

Injuries to the suprahepatic IVC are highly lethal. Vascular exclusion of the liver during operative repair is difficult particularly in the setting of massive bleeding and pre-existing shock. The procedure described herein is less invasive than direct vacular exclusion, provides hemodynamic stability through control of the aorta, and effectively controls the bleeding from the suprahepatic IVC injury through total vascular exclusion of the liver.

\section{REFERENCES}

1. Buckman RF, Miraliakbari R, Badellino MM. Juxtahepatic venous injuries: a critical review of reported management strategies. J Trauma. 2000 May;48(5):978-984.

2. Richardson JD, Franklin GA, Lukan JK, Carrillo EH, Spain DA, Miller FB, et al. Evolution in the management of hepatic trauma: a 25-year perspective. Ann Surg. 2000 Sep;232(3): 324-330.

3. Bardes JM, Grabo D, Lam L, Tadlock MD, Strumwasser A, Inaba $\mathrm{K}$. Treatment algorithm and management of retrohepatic vena cava injuries. J Trauma Acute Care Surg. 2017 Aug;83(2):340-344.

4. Schrock T, Blaisdell FW, Mathewson C. Management of blunt trauma to the liver and hepatic veins. Arch Surg. 1968 May;96 (5):698-704.

5. Burch JM, Feliciano DV, Mattox KL. The atriocaval shunt: facts and fiction. Ann Surg. 1988 May;207(5):555-568.

6. Aaron WS, Mays ET. Isolation of the retrohepatic vena cava by balloon catheter: an experimental assessment. Rev Surg. 1975 May-Jun; 32(3):222-225.

7. Pilcher DB, Harman PK, Moore EE Jr. Retrohepatic vena cava balloon shunt introduced via the sapheno-femoral junction. J Trauma. 1977 Nov;17(11):837-841.

8. Baumgartner F, Scudamore C, Nair C, Karusseit O, Hemming A. Venovenous bypass for major hepatic and caval trauma. J Trauma. 1995 Oct;39(4):671-673.

9 Biffl WL, Moore EE, Reginald F. Venovenous bypass and hepatic vascular isolation as adjunct in the repair of destructive wounds to the retrohepatic inferior vena cava. J Trauma. 1998 Aug;45(2):400-403.

10. Rogers FB, Reese J, Shackford SR, Osler TM. The use of Venovenous bypass and total vascular isolation of the liver 
in the surgical management of juxtahepatic venous injuries in blunt hepatic trauma. J Trauma. 1997 Sep;43(3):530-533.

11. Testas P, Benichou J, Benhamou M, Chanzy M. Vascular exclusion in surgery of the liver: experimental basis, technic, and clinical results. Am J Surg. 1977 Jun;133(6):692-696.

12. Coimbra R, Hoyt DB, Engelhart S, Fortlage D. Nonoperative management reduces the overall mortality of grades 3 and 4 blunt liver injuries. Int Surg. 2006 Sep-Oct;91(5):251-257.

13. Liu PP, Chen CL, Cheng YF, Hsieh PM, Tan BL, Jawan B, et al. Use of a refined operative strategy in combination with the multidisciplinary approach to manage blunt juxtahepatic venous injuries. J Trauma. 2005 Oct;59(4):940-245.

14. Khaneja SC, Pizzi WF, Barie PS, Ahmed N. Management of penetrating juxtahepatic inferior vena cava injuries under total vascular exclusion. J Am Coll Surg. 1997 May;184(5): 469-474.

15. Bisulli M. Gamberini E, Coccolini F, Scognamiglio G, Agnoletti $\mathrm{V}$. Resuscitative endovascular balloon occlusion of vena cava (REBOVC): an option in managing traumatic vena cava injuries. J Trauma Acute Care Surg. 2018 Jan;84(1)211-213.

16. Bui TD, Mills JL. Control of inferior vena cava injury using percutaneous balloon catheter occlusion. Vasc Endovascular Surg. 2009 Oct-Nov;43(5):490-493.

17. Lallemand MS, Moe DM, McClellan JM, Smith JP, Daab L, Marko S, et al. Resuscitative endovascular balloon occlusion of the aorta for major abdominal venous injury in a porcine hemorrhagic shock model. J Trauma Acute Care Surg. 2017 Aug;83(2):1054-1058.

18. Reynolds CL, Celio AC, Bridges LC, Mosquera C, O'Connell $\mathrm{B}$, Bard MR, et al. REBOA for the IVC? Resuscitative balloon occlusion of the inferior vena cava (REBOVC) to abate massive hemorrhage in retrohepatic vena cava injuries. J Trauma Acute Care Surg. 2017 Dec;83(6):1041-1046.

19. Russo RM, Williams TK, Grayson JK, Lamb CM, Cannon JW, Clement NF, et al. Extending the golden hour: partial resuscitative endovascular balloon occlusion of the aorta in a highly lethal swine liver injury model. J Trauma Acute Care Surg. 2016 Mar;80(3):372-380.

20. Ordoñez CA, Herrera-Escobar JP, Parra MW, Rodrigues-Ossa PA, Puyana JC. A severe traumatic juxtahepatic blunt venous injury. J Trauma and Acute Care Surg. 2016 Apr;80(4):674-676.

21. White JM, Cannon JW, Stannard A, Markov NP, Spencer JR, Rasmussen TE. Endovascular balloon occlusion of the aorta is superior to resuscitative thoracotomy with aortic clamping in porcine model of hemorrhagic shock. Surgery. 2011 Sep;150(3):400-409.

22. Edwards WS, Salter PP Jr., Carnaggion VA. Intraluminal aortic occlusion as a possible mechanism for controlling massive intra-abdominal hemorrhage. Surg Forum. 1953 Oct;4:496499.

23. Hughes CW. Use of intra-aortic balloon catheter tamponade for controlling intra-abdominal hemorrhage in man. Surgery. 1954 Jul;36(1):65-68.

24. Brenner ML, Moore LJ, DuBose JJ, Tyson GH, McNutt MK, Albarado RP, et al. A clinical series of resuscitative endovascular balloon occlusion of the aorta for hemorrhage control and resuscitation. J Trauma Acute Care Surg. 2013 Sep;75(3):506-511.

25. DuBose JJ, Scalea TM, Brenner M, Skiada D, Inaba K, Cannon $\mathrm{J}$, et al. The AAST prospective Aortic Occlusion for Resuscitation in Trauma and Acute Care Surgery (AORTA) registry: Data on contemporary utilization and outcomes of aortic occlusion and resuscitative balloon occlusion of the aorta (REBOA). J Trauma Acute Care Surg. 2016 Sep;81(3):409-419.

26. Gupta BK, Khaneja SC, Flores L, Eastlick L, Longmore W, Shaftan GW. The role of intra-aortic balloon occlusion in penetrating trauma. J Trauma. 1989 Jun;29(6):861-865.

27. Miura F, Takada T, Ochiai T, et al. Aortic occlusion balloon catheter technique is useful for uncontrollable massive intraabdominal bleeding after hepato-pancreato-biliary surgery. J Gastrointest Surg. 2006 Apr;10(4):519-522.

28. Moore LJ, Brenner M, Kozar RA, Pasley J, Wade CE, Baraniuk MS, et al. Implementation of resuscitative endovascular balloon occlusion of the aorta as an alternative to resuscitative thoracotomy for noncompressible truncal hemorrhage. J Trauma Acute Care Surg. 2015 Oct;79(4):523-532.

29. Doty DB, Berman JR. Control of hepatic venous bleeding by transvenous balloon catheter. Surg Gynecol Obstet. 1970 Sep;131(3):449-452.

30. McAnena OJ, Moore EE, Moore FA. Insertion of a retrohepatic vena cava balloon shunt through the saphenofemoral junction. Am J Surg. 1989 Nov;158(5):463-66.

31. Millikan JS, Moore EE, Cogbill TH, Kashuk JL. Inferior vena cava injuries - A continuing challenge. J Trauma. 1983 Mar;23(3):207-212.

32. Nicoluzzi JE, Von Bhaten LC, Laux G. Hepatic vascular isolation in treatment of a complex hepatic vein injury. J Trauma. 2007 Sep;63(3):684-686.

33. Ledgerwood AM, Kazmers M, Lucas CE. The role of thoracic aortic occlusion for massive hemoperitoneum. J Trauma. 1976 Aug;16(8):610-15.

34. Sankaran S, Lucas C, Walt AJ. Thoracic aortic clamping for prophylaxis against sudden cardiac arrest during laparotomy for acute massive hemoperitoneum. J Trauma. 1975 Apr;15(4):290-296.

35. Kralovich KA, Morris DC, Dereczyk BE, Simonetti V, Williams M, Rivers EP, et al. Hemodynamic effects of aortic occlusion during hemorrhagic shock and cardiac arrest. J Trauma. 1997 Jun;42(6):1023-1028.

37. Markov NP, Percival TJ, Morrison JJ, Ross JD, Scott DJ, Spencer JR. Physiologic tolerance of descending thoracic aortic balloon occlusion in swine model of hemorrhagic shock. Surgery. 2013 Jun;153(6):848-856.

38. Williams TK, Neff LP, Johnson MA, Ferencz SA, Davidson AJ, Russo RM, et al. Extending resuscitative endovascular balloon occlusion of the aorta: endovascular variable aortic control in a lethal model of hemorrhagic shock. J Trauma Acute Care Surg. 2016 Aug;81(2):294-301.

39. Bismuth H, Castaing D, Garden OJ. Major hepatic resection under total vascular exclusion. Ann Surg. 1989 Jul;210(1): 13-19.

40. Delva E, Barberousse JP, Nordinger B, Ollivier JM, Vacher B, Guilmet $\mathrm{C}$, et al. Hemodynamic and biochemical monitoring during major hepatic resection with use of hepatic vascular exclusion. Surgery. 1984 Mar; 95(3):309-318.

41. Offenstadt G, Huguet C, Gallot D, Bloch P. Hemodynamic monitoring during complete vascular exclusion for extensive hepatectomy. Surg Gynecol Obstet. 1978 May;146(5):709-713.

42. Bogert JN, Patel BM, Johnson DJ. Ultrasound optimization for resuscitative endovascular balloon occlusion of the aorta. J Trauma Acute Care Surg. 2017 Jan;82(1):204-207.

43. Sokol KK, Black GE, Willey SB, Kniery K, Marko ST, Eckert MJ, et al. There's an app for that: a handheld smartphonebased infrared imaging device to assess adequacy and level 
of aortic occlusion during REBOA. J Trauma Acute Care Surg. 2017 Jan;82(1)102-108.

44. Avaro JP, Mardelle V, Roch A, Gil C, de Biasi C, Oliver M, et al. Forty-minute endovascular aortic occlusion increases survival in an experimental model of uncontrolled hemorrhagic shock caused by abdominal trauma. J Trauma. 2011 Sep;71(3):720-772.

45. Evans PM, Vogt DP, Mayes JT 3rd, Henderson JM, Walsh RM. Liver resection using total vascular exclusion. Surgery. 1988 Oct;124(4):807-813.
46. van Riel WG, van Golen RF, Reiniers MJ, Heger M, van Gulik TM. How much ischemia can the liver tolerate during resection? Hepatobiliary Surg Nutr. 2016 Feb; 5(1):58-71.

47. Yellin AE, Chaffee CB, Donovan AJ. Vascular isolation in treatment of juxtahepatic venous injuries. Arch Surg. 1971 Jun;102(6):566-573.

48. Saito N, Matsumoto H, Yagi T, Hara Y, Hayashida K, Motomura T, et al. Evaluation of the safety and feasibility of resuscitative endovascular balloon occlusion of the aorta. J Trauma Acute Care Surg. 2015 May;78(5):897-903. 